Braz J Med Biol Res, October 2012, Volume 45(10) 906-912

doi: 10.1590/S0100-879X2012007500107

Silencing HIF-1 $\alpha$ reduces the adhesion and secretion functions of acute leukemia hBMSCs

Zeng Dong-Feng, Liu Ting, Chang Cheng, Zhang Xi, Liang Xue, Chen Xing-Hua and Kong Pei-Yan

The Brazilian Journal of Medical and Biological Research is partially financed by

\section{惡CNPq}

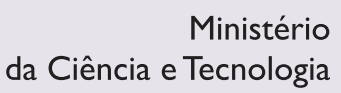

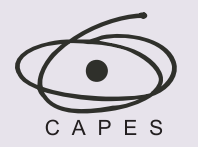

Ministério da Educação
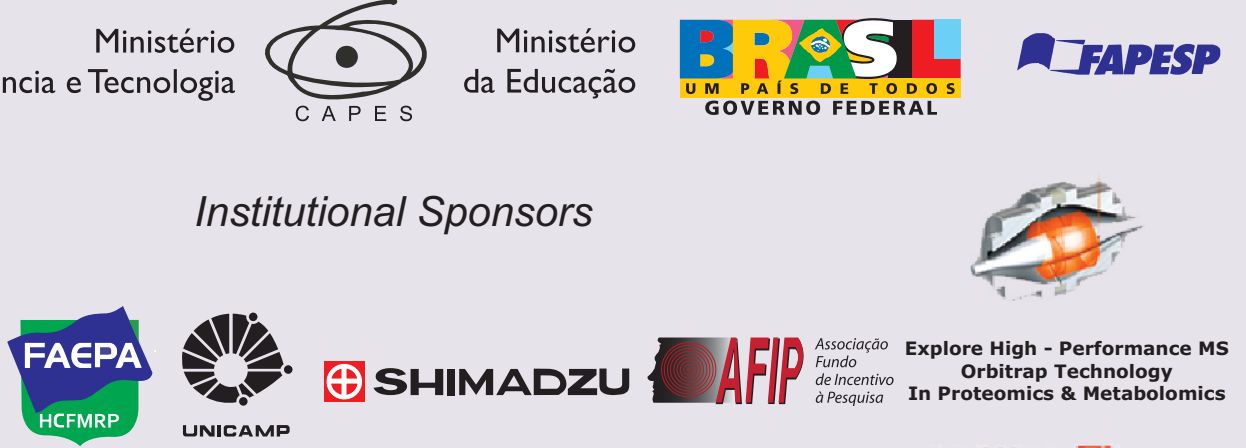

Institutional Sponsors

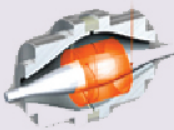

Ф SHIMADZU UNICAMP

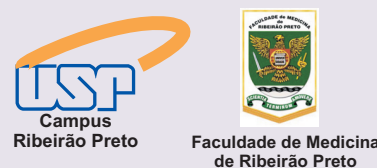

de Ribeirão Preto
deuldade de Medic 


\title{
Silencing HIF-1a reduces the adhesion and secretion functions of acute leukemia hBMSCs
}

\author{
Zeng Dong-Feng ${ }^{1 *}$, Liu Ting ${ }^{2 *}$, Chang Cheng ${ }^{1}$, Zhang $\mathrm{Xi}^{1}$, Liang Xue ${ }^{1}$, \\ Chen Xing-Hua ${ }^{1}$ and Kong Pei-Yan ${ }^{1}$
}

1Department of Hematology, XinQiao Hospital, Third Military Medical University, ChongQing, China 2Department of Ophthalmology, DaPing Hospital, Third Military Medical University, ChongQing, China

\begin{abstract}

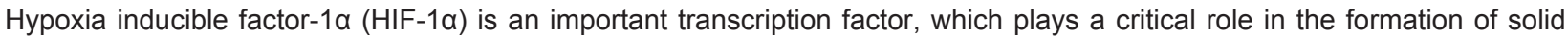
tumor and its microenvironment. The objective of the present study was to evaluate the expression and function of HIF-1 $\alpha$ in

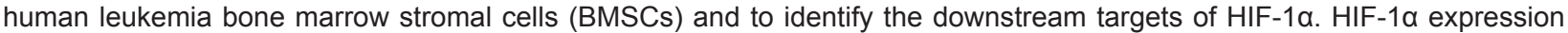
was detected at both the RNA and protein levels using real-time PCR and immunohistochemistry, respectively. Vascular endothelial growth factor (VEGF) and stromal cell-derived factor-1 $\alpha$ (SDF-1 $\alpha$ ) were detected in stromal cells by enzyme-linked immunosorbent assay. HIF-1 $\alpha$ was blocked by constructing the lentiviral RNAi vector system and infecting the BMSCs. The Jurkat cell/BMSC co-cultured system was constructed by putting the two cells into the same suitable cultured media and conditions. Cell adhesion and secretion functions of stromal cells were evaluated after transfection with the lentiviral RNAi vector of HIF-1 1 . Increased HIF-1 $\alpha$ mRNA and protein was detected in the nucleus of the acute myeloblastic and acute lymphoblastic leukemia compared with normal BMSCs. The lentiviral RANi vector for HIF-1 1 a was successfully constructed and was applied to block the expression of HIF-1a. When HIF-1a of BMSCs was blocked, the expression of VEGF and SDF-1a secreted by stromal cells was decreased. When HIF-1 $\alpha$ was blocked, the co-cultured Jurkat cell's adhesion and migration functions were also decreased. Taken together, these results suggest that HIF-1 $\alpha$ acts as an important transcription factor and can significantly affect the secretion and adhesion functions of leukemia BMSCs.
\end{abstract}

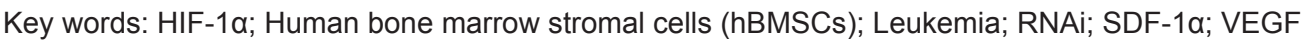

\section{Introduction}

Hypoxia-inducible factor-1 (HIF-1) is an important transcription factor associated with tumor progression and the formation of tumor stroma. It consists mainly of $\alpha$ and $\beta$ subunits, with HIF-1 $\alpha$ considered to be the major functional subunit (1). HIF-1 $\alpha$ is up-regulated in response to activation or inactivation of certain oncogenes or tumor suppressors. Rapid tissue proliferation during tumorigenesis results in severe local tissue hypoxia and leads to an imbalance between energy supply and need, thus enhancing the expression of HIF-1a $(2,3)$.

$\mathrm{HIF}-1 \alpha$ is involved in a wide range of biological functions, such as angiogenesis, cell proliferation, and cell survival, as well as vascular remodeling (4-6). Studies have suggested that the stroma of most human tumors expresses HIF-1a, which may be involved in regulating the expression of the downstream vascular endothelial growth factor (VEGF) gene, and be related to tumor angiogenesis and tumor cell migration $(7,8)$. HIF-1 $\alpha$ also plays an important role in normal hematopoiesis. HIF-1 $\alpha$ is involved in the activation of erythropoietin and thrombopoietin, which lead to the production of erythrocytes and megakaryocytes. HIF-1 $1 \alpha$ may be a key regulator affecting the migration and adhesion function of hematopoietic stem cells through the stromal cellderived factor-1/CXC receptor 4 (SDF-1/CXCR4) pathway or another pathway $(9,10)$.

As is the case for solid tumor stroma, the hematopoietic microenvironment is critical for leukemia cell adhesion and migration function. Due to the lack of solid massive tumor tissues and the tumor blood vessels that provide nutrients for the tumor cells, the roles of angiogenesis and cell hypoxia have been overlooked in the hematopoietic microenvironment of leukemia.

Correspondence: Chen Xing-Hua or Kong Pei-Yan, Department of Hematology, XinQiao Hospital, Third Military Medical University, ChongQing, China. Fax: +86-23-6520-0687. E-mail: xinghc8888@hotmail.com or peiyankong@hotmail.com

*Zeng Dong-Feng and Liu Ting contributed equally to this study.

Received January 13, 2012. Accepted May 25, 2012. Available online June 29, 2012. Published September 3, 2012. 
A recent study reported abnormal expression of $\mathrm{HIF}-1 \mathrm{a}$ in leukemia cells (11), indicating that a hypoxia-inducible environment also exists in the microenvironment of leukemia. This has led to the speculation that HIF-1a may play an important role in regulating the initiation and progression of leukemia through the hematopoietic microenvironment of the disease.

In this study, our objective was to provide evidence for the participation of HIF-1 $\alpha$ in regulating the function of the bone marrow hematopoietic microenvironment in leukemia, and to identify the possible pathway affecting the secretion and adhesion functions of leukemic bone marrow stromal cells (BMSCs).

\section{Patients and Methods}

Bone marrow samples $(N=28)$ were obtained from leukemia patients at the onset of the disease prior to chemotherapy, and control bone marrow samples were obtained from hematologically normal patients. Median age was 35 years (range $=5-61$ years). Diagnoses included acute myeloblastic leukemia without maturation (AML-M1) $(N=2)$, AML with maturation (AML-M2) $(\mathrm{N}=7)$, acute promyelocytic leukemia (AML-M3) $(\mathrm{N}=5)$, acute myelomonocytic leukemia (AML-M4) $(\mathrm{N}=3)$, acute monocytic leukemia (AML-M5) $(N=2)$, acute erythroid leukemia $(A M L-M 6)(N=2)$, and acute lymphoblastic leukemia (ALL) $(\mathrm{N}=7)$. The patients recruited for this study were diagnosed on the basis of clinical data, tissue/cell morphology, and immunophenotype. The investigation was approved by the Ethics Committee of XinQiao Hospital, and all patients gave written informed consent to participate.

\section{Human BMSC (hBMSC) culture}

Heparinized bone marrow aspirates (2-3 mL) were diluted 1:1 in phosphate-buffered saline (PBS) followed by centrifugation at $550 \mathrm{~g}\left(20^{\circ} \mathrm{C}, 5 \mathrm{~min}\right)$. The supernatants were discarded and the pellets were resuspended in $4 \mathrm{~mL}$ PBS. Bone marrow mononuclear cells were separated by Ficoll density gradient centrifugation and washed twice in PBS. The cells were seeded in tissue culture flasks in DMEM-F12 medium containing $10 \%$ fetal calf serum (FCS) and incubated at $37^{\circ} \mathrm{C}$ in $5 \% \mathrm{CO}_{2}$. Upon confluence, the cells were harvested using Trypsin, centrifuged, and resuspended in DMEM-F12 medium containing 10\% FCS.

\section{Real-time PCR}

Real-time quantitative PCR was performed using the Bio-Rad icycler PCR Detection System (Bio-Rad, France). Ready-made primers and TaqMan probes for HIF-1 $\alpha$ and GAPDH were purchased from TaKaRa Bio Company (Japan). PCR was performed in a total volume of $25 \mu \mathrm{L}$, containing $0.3 \mu \mathrm{m} / \mathrm{L}$ of each Taqman probe, $0.4 \mu \mathrm{m} / \mathrm{L}$ of each primer, 1X Taq buffer from SYBR ${ }^{\circledR}$ Green Real-Time PCR Master Mix (Toyobo, Japan), and $10 \mathrm{ng}$ sample
cDNA or various amounts $(0.001,0.01,0.1,1$, or $10 \mathrm{ng})$ of standard cDNA. Total hBMSC RNAs were extracted using Trizol reagent. Each test and standard cDNA was amplified in three different wells. Cycling conditions were as follow: $3 \mathrm{~min}$ at $95^{\circ} \mathrm{C}, 40$ cycles of $30 \mathrm{~s}$ at $93^{\circ} \mathrm{C}, 30 \mathrm{~s}$ at $60^{\circ} \mathrm{C}, 1$ min at $72^{\circ} \mathrm{C}$, and an additional $5 \mathrm{~min}$ at $72^{\circ} \mathrm{C}$. Data were analyzed using the Bio-Rad icycler iq sequence detection software according to manufacturer instructions. Threshold cycle number was determined, and the starting gene copy number relative to the reference was determined for each well using a standard curve. The normalized gene expression level is reported as the ratio between the mean value of the HIF-1a gene and the GAPDH gene in each sample. Forward $(F)$ and reverse $(R)$ primers were designed using the Beacon Designer software (Bio-Rad). Primers for HIF-1a were F: 5'-CCAGACGATCATGCAGCTAC-3'; R: 5'-ggtttctgctgccttgtatag-3', and for GAPDH were F: 5'-CCATCAATGACCCCTTCATTG-3'; R: 5'-CATGGGTGG AATCATATTGGAAC-3' (11).

\section{Immunohistochemical analysis}

The streptavidin-peroxidase technique and 3,3-diaminobenzidine (DAB) staining were used for immunohistochemical analysis. Briefly, the following protocol was used: samples were dewaxed and hydrated, followed by removal of endogenous peroxidase with $3 \%$ hydrogen peroxide. Antigen restoration was carried out in a microwave oven in citrate-buffered saline. After blocking with normal goat serum, the samples were incubated with anti-human HIF-1a antibody, using PBS as a negative control. Incubations were carried out overnight at $4^{\circ} \mathrm{C}$, followed by incubation with biotinylated goat-anti-mouse IgG for $10 \mathrm{~min}\left(\right.$ at $18-20^{\circ} \mathrm{C}$ ), and conjugation and incubation with peroxidase-labeled streptavidin for $10 \mathrm{~min}$ (at $18-20^{\circ} \mathrm{C}$ ), staining with $\mathrm{DAB}$, washing with water, and finally mounting the coverslip with neutral resin. Positivity was evaluated according to the extent and intensity of antibody staining using peroxidase-labeled streptavidin for $10 \mathrm{~min}\left(18-20^{\circ} \mathrm{C}\right)$, development with $\mathrm{DAB}$, counterstaining with hematoxylin, and finally mounting the coverslip with neutral resin (12).

\section{Western blot analysis}

Proteins were extracted in ice-cold modified radioimmunoprecipitation assay buffer. Protein concentrations were measured by the method of Bradford according to manufacturer instructions (Bio-Rad). Equal amounts of protein $(40 \mu \mathrm{g})$ were separated on a $10 \%$ SDS gel and blotted onto a nitrocellulose membrane. HIF-1 $\alpha$ was detected by incubating with mouse anti-HIF-1 $\alpha$ antibodies overnight $\left(4^{\circ} \mathrm{C}\right)$, followed by incubation with horseradish peroxidase-conjugated anti-mouse IgG. Relative expression was determined by densitometry and analyzed by the Quality One software from Bio-Rad (USA). An anti-GAPDH antibody was used as a loading control. 


\section{Construction of the recombinant miR-HIF-1a lentivirus vector}

Three pairs of micro-RNA (miRNA) primers were designed online using the software provided by the website (www.invitrogen.com/rnai), and then synthesized by Invitrogen. The pcDNA 6.2-GW/EmGFP-miR-HIF-1 $\alpha$ plasmid was constructed following manufacturer instructions using the Block-iT Pol II miR RNAi Expression Vector Kit. The construct was verified by sequencing. The primers used for sequencing were: EmGFP forward sequencing primer: 5'-GGCATGG ACGAGCTGTACAA-3' and miRNA reverse sequencing primer: 5'-CTCTAGATC AACCACTTTGT-3'.

The effect of RNAi was confirmed by real-time PCR and the second pair of primers was then chosen for the miRNA target. PcDNA 6.2-GW/EmGFP-miR-HIF-1a maxiprep was performed using the PureLink HiPure endotoxin-free plasmid maxiprep kit. BP/LR reaction was performed following manufacturer instructions using the Gateway cloning system. The recombinant vector was treated with proteinase $\mathrm{K}$ and used to transform One Shot Stbl $3^{\mathrm{TM}}$ competent cells. Several positively selected colonies were picked for miniprep and sent to Invitrogen for sequencing to confirm if the insert had been successfully integrated into the pLenti6/ V5-GW/EmGFP-HIF-1a-miR plasmid. The primers used for sequencing were CMV forward: 5'-CGCAAATGGGCGG TAGGCGTG-3' and V5 (C-term) reverse: 5'-ACCGAGG AGAGGGTTAGGGAT-3'.

\section{Lentivirus packaging, titer determination and infection}

The recombinant pLenti6/V5-GW/EmGFP-HIF-1amiR plasmid DNA and the lentiviral packaging mix were diluted in serum-free Opti-MEM I medium, mixed gently, and used to transfect $6 \times 10^{6} 293 \mathrm{FT}$ cells using the Lipofectamine 2000 transfection reagent. The supernatants were collected and centrifuged to harvest the viruses. Once the 3T3 cells on 6-well plates used for titration reached $30-50 \%$ confluence, the virus suspensions, diluted from $10^{-7}$ to $10^{-2}$ with the addition of polybrene (final concentration, $6 \mu \mathrm{g} / \mathrm{mL}$ ), were added to fresh complete medium containing blasticidin (final concentration, 2.0 $\mu \mathrm{g} / \mathrm{mL}$ ) and incubated overnight at $37^{\circ} \mathrm{C}$ in a humidified $5 \% \mathrm{CO}_{2}$ incubator. The titers were determined by flow cytometry 4 days post-transduction and the EmGFP lentivirus titers were calculated based on the dilutions at which the percentage of EmGFP-positive cells fell within the range of $1-30 \%$. The following formula was used to calculate the titer: $[F \times C / V] \times D$, where $F=$ percentage of green fluorescent protein (GFP)-positive cells; $C=$ total number of cells in the well at the time of transduction; $\mathrm{V}=$ volume of inoculum $(\mathrm{mL}) ; \mathrm{D}=$ fold dilution of lentivirus. The multiplicity of infection value was determined before transduction of target cells. The target cells were transduced with appropriately diluted virus supernatants when they reached $30-50 \%$ confluence on 6 -well plates, and polybrene (final concentration, $6 \mu \mathrm{g} / \mathrm{mL}$ ) was added to the wells. Blasticidin (final concentration $2.0 \mu \mathrm{g} / \mathrm{mL}$ ) was used to select stably transduced cells.

\section{ELISA}

Supernatants were collected when the hBMSCs were approximately $80 \%$ confluent. The expression levels of VEGF and SDF-1 $\alpha$ were detected using VEGF and SDF-1a ELISA kits according to manufacturer recommendations.

\section{Adhesion of Jurkat cells to human stromal cells}

Acute leukemia hBMSCs were harvested by trypsinization and seeded at $2 \times 10^{4}$ cells per well on 24 -well plates. The cells were divided into three groups: miR RNAi lentiviral infection group, miR RNAi-neg lentiviral infection group, and non-infection group. Jurkat cells were seeded above the stromal cell layer when the hBMSCs reached approximately $80 \%$ confluence, and the two cell lines were then co-cultured for $24 \mathrm{~h}$. Percent adhesion was calculated using the following formula: $\%$ adhesion = Jurkat cells adhered to stromal cells / total Jurkat cells $\times 100 \%$.

\section{Transwell migration assay}

Stromal cells were digested with $0.25 \%$ Trypsin, the cell density was adjusted to $1 \times 10^{5} / \mathrm{mL}$ and the cells were inoculated onto 24-well plates (5 wells/sample). Upon reaching $70-80 \%$ confluence, the culture medium was replaced with fresh medium $(800 \mu \mathrm{L} /$ well). Transwell inserts coated with matrigel pre-hydrated for $2 \mathrm{~h}$ were placed in the wells. Jurkat cells $\left(1 \times 10^{6} / \mathrm{mL}\right)$ were seeded in the upper chamber ( $400 \mu \mathrm{L} /$ well) and incubated for a further $24 \mathrm{~h}$. The inserts were then removed and the cells on the insert wall and surface were wiped off using cotton swabs soaked in serum-free RPMI 1640 medium. The cells were subjected to Wright's staining and observed under a microscope at 200X magnification. Septum-permeating cells were counted in five random visual fields. The number of septum-permeating cells was used to express the migration capacity of the Jurkat cells.

\section{Statistical analysis}

Differences between two groups were compared using $t$-tests. Values of $\mathrm{P}<0.05$ were considered to be statistically significant.

\section{Results}

\section{High expression of HIF-1a in stromal cells from acute leukemia patients}

HIF-1 a mRNA was detected by real-time PCR. HIF-1 $\alpha$ levels were higher in the AML $(0.83 \pm 0.11)$ and ALL $(0.71$ $\pm 0.06)$ groups than in the normal controls $(0.30 \pm 0.13$; $P<$ 0.01). Immunohistochemical analysis showed that the HIF-1a 


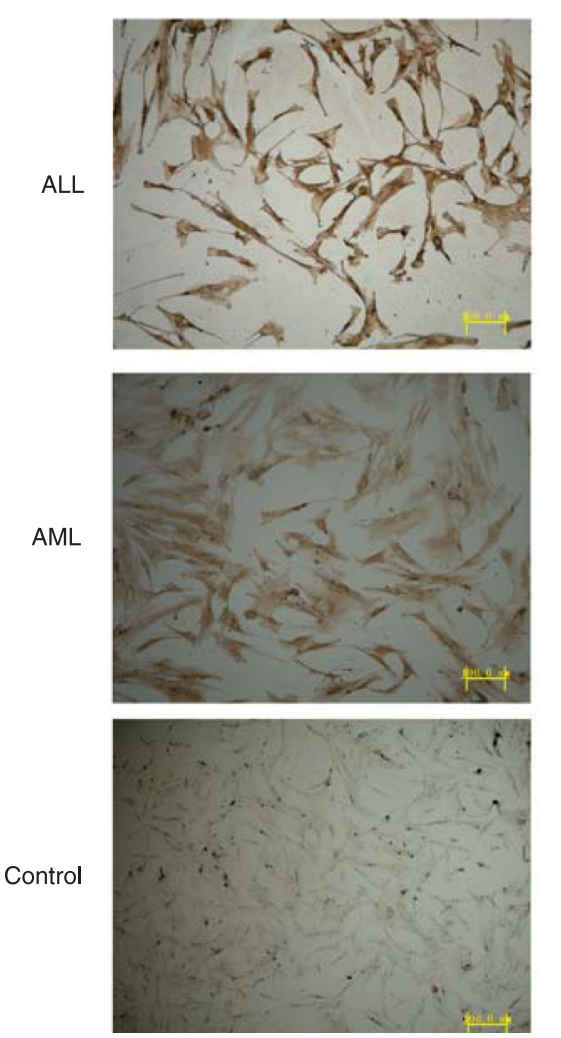

Figure 1. HIF-1a protein expression detected by immunohistochemistry. The HIF-1 $\alpha$-positive cells are shown stained brown. The levels of HIF-1 $1 \alpha$ protein in the acute myeloblastic leukemia $(\mathrm{AML})$ and acute lymphoblastic leukemia (ALL) groups were higher than in the normal control $(P<0.01, t$-test $)$.

protein was localized mainly in the nucleus and partly in the cytoplasm of the stromal cells (Figure 1).

HIF-1 1 mRNA and protein expression were successfully silenced by the constructed plasmid

Sequencing results showed that the third set of primers (forward 5'-TGCTGAGGACTAGGTGTCTGATCCTG GTTTTGGCCACTGACTGACCAGGATCACACCTAGT CCT-3', reverse 5'-CCTGAGGACTAGGTGTGATCCTG GTCAGTCAGTGGCCAAAACCAGGATCAGACACCTA GTCCTC-3') were successfully inserted into the cloning vector pcDNA 6.2-GW/EmGFP-miR and were successfully inserted into the lentiviral expression vector pLenti6/ V5-GW/EmGFP-miR. Virus was successfully produced using the pLenti6/V5-GW/EmGFP-HIF-1a-miR construct and was used to infect stromal cells. The EmGFP protein was visible under a fluorescence microscope (Figure 2). Real-time PCR and Western blot were performed after 4 days of incubation to evaluate the HIF-1a knockdown efficiency. The HIF-1 $\alpha$ mRNA and protein levels in the infected stromal cells were much lower than that in the pre-RNAi cells (Figures 3 and 4).
A

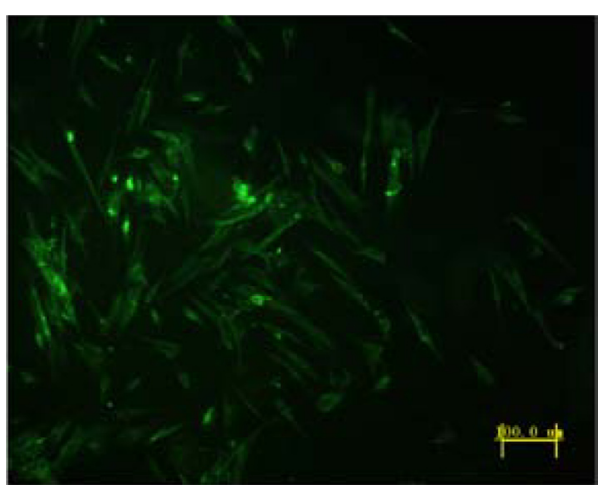

B

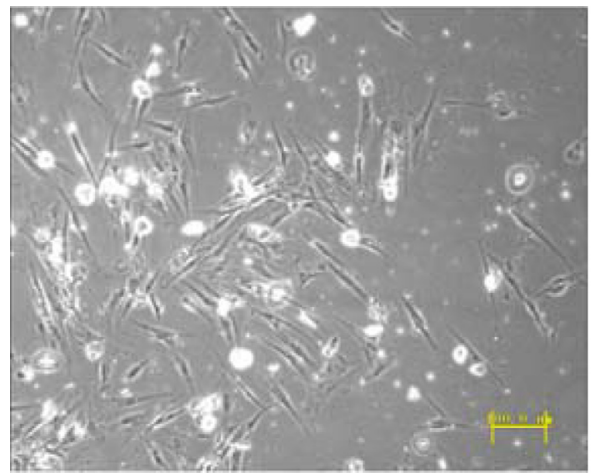

Figure 2. $A$, The successfully infected stromal cells emitted green fluorescence under a fluorescence microscope. The percentage of EmGFP-positive cells was $80-90 \%$. B, Normal light microscopy.

\section{HIF-1 $\alpha$-silenced stromal cells expressed low levels of SDF- $1 \alpha$ and VEGF}

According to ELISA analysis, after HIF-1a silencing, the level of the SDF-1 $\alpha$ protein decreased from 4316.25 \pm 582.22 to $3456.98 \pm 231.16 \mathrm{pg} / \mathrm{mL}$ in the ALL group ( $P$ $<0.05$ ), and from $5885.94 \pm 422.23$ to $3965.48 \pm 612.78$ $\mathrm{pg} / \mathrm{mL}$ in the AML group $(\mathrm{P}<0.05)$. The expression of the VEGF protein decreased from $296.21 \pm 65.38$ to $185.88 \pm$ $58.21 \mathrm{pg} / \mathrm{mL}$ in the ALL group $(P<0.05)$ and from $435.68 \pm$ 73.53 to $262.25 \pm 35.89 \mathrm{pg} / \mathrm{mL}$ in the AML group $(\mathrm{P}<0.05)$. These data suggest that HIF-1 $\alpha$ may act as a transcription factor for SDF-1 $\alpha$ and VEGF, and that down-regulation of HIF-1 $\alpha$ can directly affect the expression of SDF-1 $\alpha$ and VEGF (Figure 5).

\section{Jurkat cell adhesion and migration decreased after HIF-1 $\alpha$ silencing in co-cultured hBMSCs}

Jurkat cells were divided into three groups: i) cells co-cultured with miR RNAi hBMSCs, ii) cells co-cultured with miR-neg hBMSCs, and iii) cells co-cultured with noninfected hBMSCs. The cell migration rate was much lower in group i $(28.35 \pm 5.92 \%)$ than in group ii $(50.34 \pm 7.82 \%)$, and group iii $(47.58 \pm 6.63 \%$; $P<0.05)$.

The number of septum-permeating cells in the Transwell migration assay was also much lower in group i ( $5 \pm 8$ cells) 


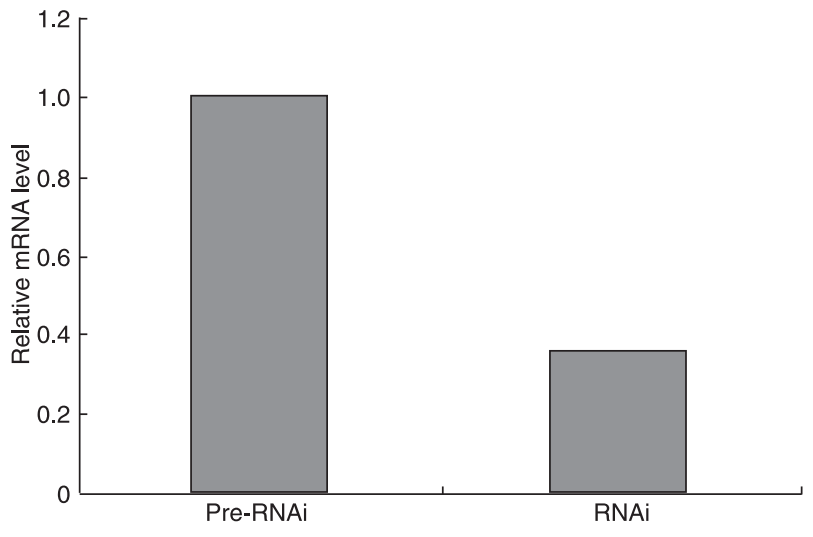

Figure 3. The effect of RNAi on HIF-1a expression examined by real-time RT-PCR. Analysis revealed that the levels of HIF1a mRNA were significantly down-regulated by $65 \%$ in the RNAi group compared to the pre-RNAi group ( $\mathrm{P}<0.01, t$-test).

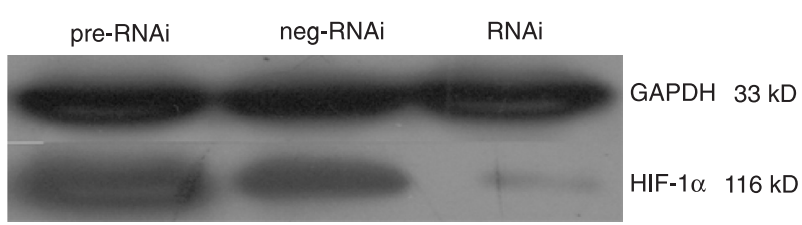

Figure 4. The stromal cells in the RNAi group expressed a lower level of the hypoxia-inducible factor-1 $\alpha$ (HIF-1 $\alpha$ ) protein (average gray value $=0.336 \pm 0.053$ ) than in the pre-RNAi group (average gray value $=0.845 \pm 0.084 ; \mathrm{P}<0.05$ ) and the neg-RNAi group (average gray value $=0.798 \pm 0.079 ; P<0.05$ ) at 4 days after infection, indicating that the RNAi lentivirus vector targeting HIF$1 \alpha$ could efficiently knock down the protein expression of HIF-1 $1 \alpha$. The $t$-test was used for statistical analyses.

than in group ii $(45 \pm 12)$ or group iii $(48 \pm 9$; $P<0.05)$.

The results reported above showed that after downregulating the expression of HIF-1a, the rate of migration and adhesion of co-cultured Jurkat cells was decreased. These results suggest that the HIF-1 $1 \alpha$ signaling pathway could significantly affect the adhesion capacity of the stromal cells, and indirectly influence the migration ability of the co-cultured Jurkat cells, either by endocrine or other mechanisms.

\section{Discussion}

The abnormal bone marrow microenvironment is the most important event in the initiation and progression of leukemia. Previous studies have shown that a variety of morphological and functional abnormalities exist in the leukemia bone marrow microenvironment, including significantly increased microvascular density, increased expression of some vascular-related growth factors such as VEGF, and some chemokines such as SDF-1 $(10,13,14)$. The disturbance of the leukemia bone marrow microenviron-
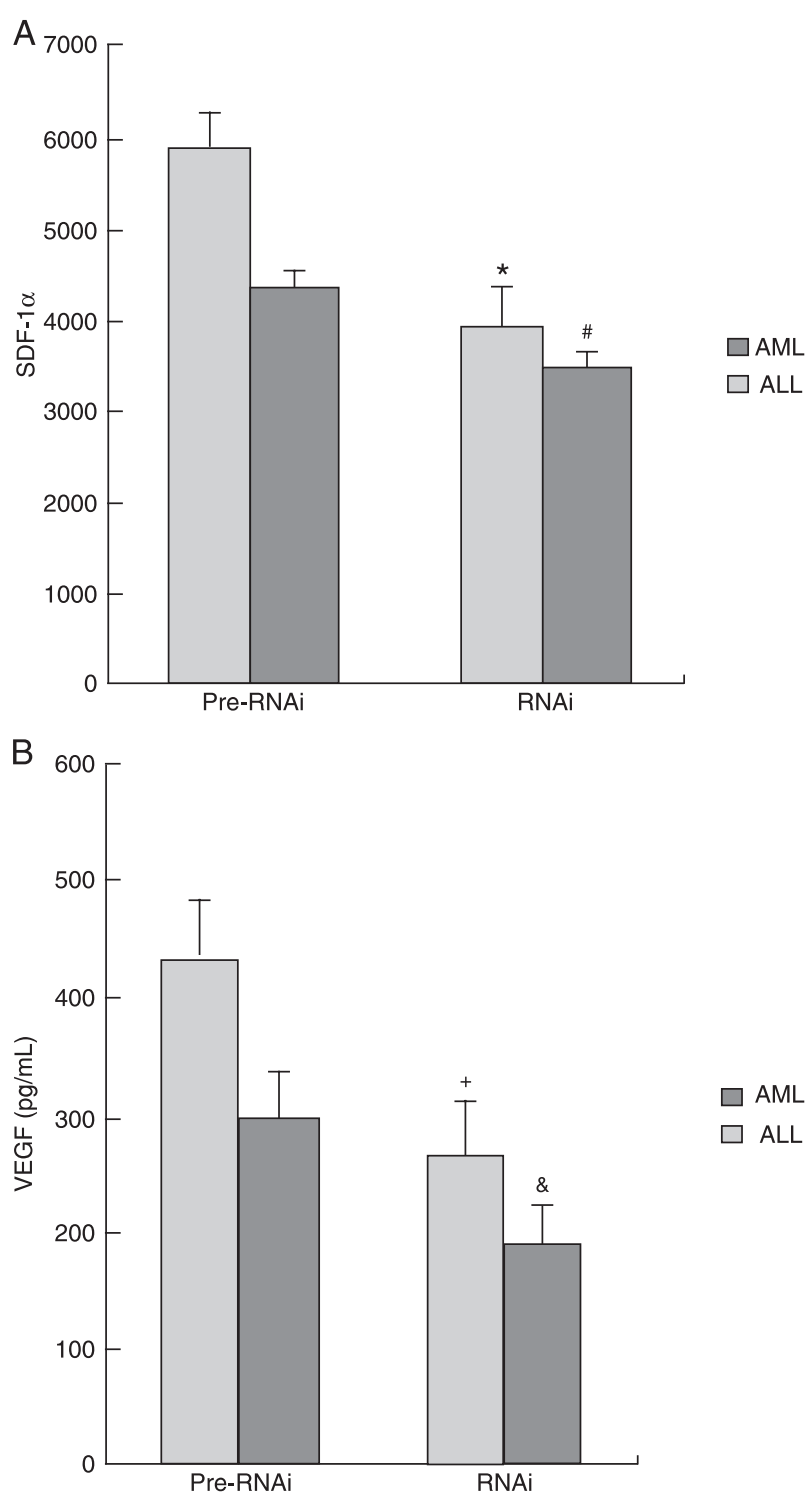

Figure 5. Changes in VEGF and SDF-1 protein expression after HIF-1a silencing in stromal cells. $A$, SDF-1a expression in stromal cells decreased in both the AML ( ${ }^{*} P<0.01$, Pre-RNAi vs RNAi group) and ALL (\#P<0.01, Pre-RNAi vs RNAi group) groups after HIF-1 $\alpha$ silencing. $B$, VEGF protein expression in stromal cells decreased in both the AML $\left({ }^{+} \mathrm{P}<0.01\right.$, Pre-RNAi vs RNAi group) and ALL ( $\& P<0.01$, Pre-RNAi vs RNAi group) groups after HIF$1 \alpha$ silencing. The $t$-test was used for statistical analyses. VEGF $=$ vascular endothelial growth factor; SDF-1 $=$ stromal cell-derived factor-1; HIF-1 $\alpha$ = hypoxia-inducible factor-1; AML = acute myeloblastic leukemia; $A L L$ = acute lymphoblastic leukemia.

ment could promote the production of abnormal adhesion molecules and chemokines, which in turn could promote the adhesion and colonization of leukemia cells to the "niches" of the local microenvironment, thus allowing the leukemia cells to evade chemotherapy through the shielding role of 
the local hematopoietic matrix.

Recent studies have shown that HIF-1a is widely found in all histiocytes, as well as in peripheral leukocytes $(3,15,16)$. Moreover, increased HIF-1 $1 \alpha$ expression occurs in a variety of solid tumor cells and stromal cells, which may be related to their migration and adhesion $(17,18)$. In the current study, HIF-1 $\alpha$ mRNAand protein expression in acute leukemia hBMSCs were significantly increased compared to control, suggesting that HIF-1a may have been involved in the formation of the leukemia bone marrow hypoxic microenvironment. Studies on solid tumors found that the regulatory axis of $\mathrm{HIF}-1 \alpha$ transcription comprised a variety of downstream factors, including VEGF, while studies on the hematopoietic microenvironment of leukemia found high levels of expression of VEGF and SDF-1 $(1,19,20)$. In the current study, we found that VEGF and SDF-1 expression levels were increased in acute leukemia hBMSCs. The levels were significantly reduced after HIF-1a silencing, but remained much higher than the control levels. These results suggest that HIF-1a may be involved in the regulation of VEGF and SDF-1 expression in the bone marrow microenvironment of leukemia.

The adhesion of leukemia cells to hBMSCs and their shielding by these cells may be key factors in determining the drug resistance and relapse of leukemia. The SDF-1/ CXCR4 axis plays an important role in the adhesion and migration of leukemia cells to the bone marrow matrix. SDF-1 induces chemotaxis of various hematopoietic cells along its concentration gradient, and also regulates the activity of extracellular signal-regulated kinase through the SDF-1/CXCR4 signaling system, which directly affects the integrin-mediated cell adhesion $(21,22)$. In the present study, we found that the adhesion rate of Jurkat cells was significantly reduced when they were co-cultured with HIF-1 $1 \alpha$-silenced acute leukemia hBMSCs compared to coculture with normal hBMSCs, suggesting that HIF-1a may influence the adhesion capacity of hBMSCs to leukemia cells by regulating SDF-1 expression.

HIF-1 $1 \alpha$ has been proposed as an important marker of infiltration and metastasis of solid tumors. Helczynska et al. (23) reported that the expression of HIF-1a in breast cancer increased significantly in the cell layers surrounding the central necrosis and on the margin of tumor infiltration, as well as around tumor vessels, while no HIF-1 $\alpha$ expression was detected in the normal tissues around the tumors. It has also been suggested that HIF-1 1 a might be

\section{References}

1. Adams JM, Difazio LT, Rolandelli RH, Lujan JJ, Hasko G, Csoka B, et al. HIF-1: a key mediator in hypoxia. Acta Physiol Hung 2009; 96: 19-28.

2. Doe MR, Ascano JM, Kaur M, Cole MD. Myc posttranscriptionally induces HIF1 protein and target gene expression in involved in the expression of tumor infiltration factors, including matrix metalloproteinase and cathepsin $D$ (23-25). Leukemia cells, like solid tumor cells, also have the ability to migrate and infiltrate. Previous studies found that the migration and homing of leukemia cells to myeloid bone marrow in vivo mainly relied on the chemotactic influence of chemokines on the hematopoietic microenvironment $(26,27)$. Binding of a chemokine to its receptor leads to the formation of a chemokine/receptor complex. This complex then activates second messengers including $\mathrm{Ca}^{2+}$ and phosphatidylinositol 3-kinase, which can in turn activate downstream protein kinases involved in the regulation of cell adhesion- and migration-related proteins. Chemotaxis will then be initiated as in solid tumors (28). Leukemia cells that colonize niches formed by the bone marrow stromal endothelium could effectively avoid chemotherapeutic toxicity, thus representing 'seeds' for future relapse and minimal residual disease.

In the current study, we used the Transwell insert system to determine the impact of HIF-1a in acute leukemia stromal cells on the migration and infiltration ability of cocultured Jurkat cells. The migration capacity of Jurkat cells induced by acute leukemia hBMSCs decreased markedly after silencing of HIF-1 $\alpha$ gene expression. Thus, inhibition of HIF-1a gene expression could significantly reduce the possibility of residual leukemia formation. The mechanism whereby HIF-1 1 a silencing affects the migration and infiltration capacity of Jurkat cells may be related to the expression of chemokines, which are regulated by HIF-1a.

The results of the present study indicate that an HIF1a/VEGF/SDF-1 signaling pathway might exist in leukemic hBMSCs and that HIF-1 $\alpha$ might play a role in transforming the bone marrow microenvironment during the initiation and progression of leukemia. These results suggest that the HIF-1 $\alpha / V E G F / S D F-1$ signaling pathway may represent a potential therapeutic target in acute leukemias.

\section{Acknowledgments}

Research supported in part by the Military Natural Science Fund of China (\#06-MB238), University Natural Science Fund of the Third Military Medical University (\#2007XG33), Natural Science fund of Chongqing (\#2009BB5151), and National Natural Science Foundation of China (\#81000195). normal and cancer cells. Cancer Res 2012; 72: 949-957.

3. Hede K. Low-dose anthracyclines may block HIF-1 and stop tumor growth. J Natl Cancer Inst 2009; 101: 368-370.

4. Monti E, Gariboldi MB. HIF-1 as a target for cancer chemotherapy, chemosensitization and chemoprevention. Curr Mol 
Pharmacol 2011; 4: 62-77.

5. Doublier S, Belisario DC, Polimeni M, Annaratone L, Riganti C, Allia E, et al. HIF-1 activation induces doxorubicin resistance in MCF7 3-D spheroids via P-glycoprotein expression: a potential model of the chemo-resistance of invasive micropapillary carcinoma of the breast. BMC Cancer 2012; 12: 4 .

6. Comerford KM, Wallace TJ, Karhausen J, Louis NA, Montalto MC, Colgan SP. Hypoxia-inducible factor-1-dependent regulation of the multidrug resistance (MDR1) gene. Cancer Res 2002; 62: 3387-3394.

7. Zhong H, Mabjeesh N, Willard M, Simons J. Nuclear expression of hypoxia-inducible factor 1 alpha protein is heterogeneous in human malignant cells under normoxic conditions. Cancer Lett 2002; 181: 233-238.

8. Jensen RL, Ragel BT, Whang K, Gillespie D. Inhibition of hypoxia inducible factor-1alpha (HIF-1alpha) decreases vascular endothelial growth factor (VEGF) secretion and tumor growth in malignant gliomas. J Neurooncol 2006; 78: 233-247.

9. Keswani SC, Bosch-Marce M, Reed N, Fischer A, Semenza GL, Hoke A. Nitric oxide prevents axonal degeneration by inducing HIF-1-dependent expression of erythropoietin. Proc Natl Acad Sci U S A 2011; 108: 4986-4990.

10. Takubo K, Goda N, Yamada W, Iriuchishima H, Ikeda E, Kubota Y, et al. Regulation of the HIF-1alpha level is essential for hematopoietic stem cells. Cell Stem Cell 2010; 7: 391-402.

11. Xiong YS, Zhou YH, Rong GH, Wu WL, Liang Y, Yang ZX, et al. Siglec-1 on monocytes is a potential risk marker for monitoring disease severity in coronary artery disease. Clin Biochem 2009; 42: 1057-1063.

12. Stroka DM, Burkhardt T, Desbaillets I, Wenger RH, Neil DA, Bauer C, et al. HIF-1 is expressed in normoxic tissue and displays an organ-specific regulation under systemic hypoxia. FASEB J 2001; 15: 2445-2453.

13. Gibbs BF, Yasinska IM, Oniku AE, Sumbayev VV. Effects of stem cell factor on hypoxia-inducible factor 1 alpha accumulation in human acute myeloid leukaemia and LAD2 mast cells. PLoS One 2011; 6: e22502.

14. de Oliveira CE, Cavassin GG, Perim AL, Nasser TF, de Oliveira KB, Fungaro $\mathrm{MH}$, et al. Stromal cell-derived factor- 1 chemokine gene variant in blood donors and chronic myelogenous leukemia patients. J Clin Lab Anal 2007; 21: 49-54.

15. Takahashi Y, Nishikawa M, Takakura Y. Inhibition of tumor cell growth in the liver by RNA interference-mediated suppression of HIF-1alpha expression in tumor cells and hepatocytes. Gene Ther 2008; 15: 572-582.

16. Kong T, Eltzschig HK, Karhausen J, Colgan SP, Shelley CS. Leukocyte adhesion during hypoxia is mediated by HIF-1dependent induction of beta2 integrin gene expression. Proc Natl Acad Sci U S A 2004; 101: 10440-10445.
17. Eckerich C, Zapf S, Fillbrandt R, Loges S, Westphal M, Lamszus K. Hypoxia can induce c-Met expression in glioma cells and enhance SF/HGF-induced cell migration. Int $J$ Cancer 2007; 121: 276-283.

18. Fujiwara S, Nakagawa K, Harada H, Nagato S, Furukawa K, Teraoka M, et al. Silencing hypoxia-inducible factor-1alpha inhibits cell migration and invasion under hypoxic environment in malignant gliomas. Int J Oncol 2007; 30: 793-802.

19. Simiantonaki N, Jayasinghe $C$, Michel-Schmidt R, Peters K, Hermanns MI, Kirkpatrick CJ. Hypoxia-induced epithelial VEGF-C/VEGFR-3 upregulation in carcinoma cell lines. Int $J$ Oncol 2008; 32: 585-592.

20. Pegahi R, Poyer F, Legrand E, Cazin L, Vannier JP, Lamacz M. Spontaneous and cytokine-evoked production of matrix metalloproteinases by bone marrow and peripheral blood pre-B cells in childhood acute lymphoblastic leukaemia. Eur Cytokine Netw 2005; 16: 223-232.

21. Winter SS, Sweatman JJ, Lawrence MB, Rhoades TH, Hart $A L$, Larson RS. Enhanced T-lineage acute lymphoblastic leukaemia cell survival on bone marrow stroma requires involvement of LFA-1 and ICAM-1. Br J Haematol 2001; 115: 862-871.

22. Arai A, Jin A, Yan W, Mizuchi D, Yamamoto K, Nanki T, et al. SDF-1 synergistically enhances IL-3-induced activation of the Raf-1/MEK/Erk signaling pathway through activation of Rac and its effector Pak kinases to promote hematopoiesis and chemotaxis. Cell Signal 2005; 17: 497-506.

23. Helczynska K, Kronblad A, Jogi A, Nilsson E, Beckman S, Landberg G, et al. Hypoxia promotes a dedifferentiated phenotype in ductal breast carcinoma in situ. Cancer Res 2003; 63: 1441-1444

24. Koga F, Tsutsumi S, Neckers LM. Low dose geldanamycin inhibits hepatocyte growth factor and hypoxia-stimulated invasion of cancer cells. Cell Cycle 2007; 6: 1393-1402.

25. Ceradini DJ, Kulkarni AR, Callaghan MJ, Tepper OM, Bastidas N, Kleinman ME, et al. Progenitor cell trafficking is regulated by hypoxic gradients through HIF-1 induction of SDF-1. Nat Med 2004; 10: 858-864.

26. Monaco G, Belmont JW, Konopleva M, Andreeff M, Tavor $\mathrm{S}$, Petit I, et al. Correlation between CXCR4 and homing or engraftment of acute myelogenous leukemia. Cancer Res 2004; 64: 6832-6833.

27. Gazitt Y. Homing and mobilization of hematopoietic stem cells and hematopoietic cancer cells are mirror image processes, utilizing similar signaling pathways and occurring concurrently: circulating cancer cells constitute an ideal target for concurrent treatment with chemotherapy and antilineage-specific antibodies. Leukemia 2004; 18: 1-10.

28. Chien W, Yin D, Gui D, Mori A, Frank JM, Said J, et al. Suppression of cell proliferation and signaling transduction by connective tissue growth factor in non-small cell lung cancer cells. Mol Cancer Res 2006; 4: 591-598. 